\title{
Natural Human-Robot Interaction
}

\author{
Takayuki Kanda \\ ATR Intelligent Robotics \& Communication Labs \\ Kyoto 619-0288, Japan
}

There are a lot of human-like robots developed recently. Researchers have started to explore the way to make human-like robots interacts with humans in a similar way as humans do, that is, natural human-robot interaction (HRI). We have faced the lack of knowledge on human behavior in interaction. There are existing literatures for modeling humans' information processing in conscious, such as about language understanding, and generation of utterance and gesture. However, when we try to build a model of natural interaction for robots, we faced a difficulty from the fact that natural interaction involves a lot of humans' unconscious information processing. Without much conscious efforts, people communicate with each other with naturally using body properties, such as gaze and gestures; but without explicit model of thorough information processing, we cannot build robots that engage in natural HRI. This talk introduces a series of studies for modeling human behavior for natural human-robot interaction. Built on existing knowledge in psychology and cognitive science, we have started to build models where observation of people's behavior were also contributed a lot. We have modeled behaviors for robots for interacting with people as well as for behaving in an environment. Moreover, we have also explored a case where behavior in interaction is inter-related with environments.

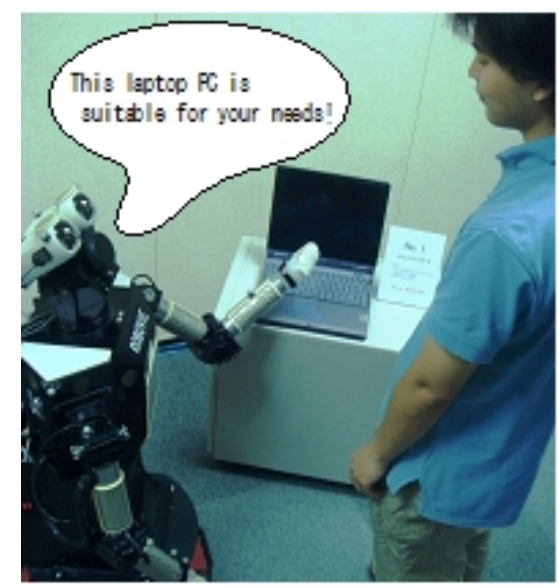

Fig. 1. A scene of interaction with a robot 